\title{
Steering the Mechanism of the Furan-Maleimide Retro-Diels-Alder Reaction to a Sequential Pathway with an External Mechanical Force
}

\author{
Manuel Cardosa-Gutierrez, ${ }^{1}$ Guillaume De Bo, ${ }^{2}$ Anne-Sophie Duwez, ${ }^{1}$ Françoise Remacle ${ }^{1}$ \\ ${ }^{1}$ UR Molecular Systems, Department of Chemistry, University of Liège, 4000 Liège, \\ Belgium \\ ${ }^{2}$ Department of Chemistry, University of Manchester, Manchester M13 9PL, UK
}

\begin{abstract}
(max 250 words)
Mechanical forces are known to control rates of chemical reactions and govern reaction pathways, possibly inducing a change of mechanism with respect to the zero force one. We report on a switching of mechanism of the retro Diels-Alder bond breaking from concerted at zero force to sequential under tension for four furan-maleimide adducts, mechanophores widely used in polymer mechanochemistry because they can undergo reversible breakage under tension. The four different adducts differ by their regio- and stereochemistry. The reaction paths on the force modified potential energy surfaces were characterized by isometric and isotensional approaches and determining stationary points (equilibrium geometries and transition states) as a function of the applied force, as well as by analyzing the redistribution of strain energy over the internal degrees of freedom. We evidence different bond breaking pathways and rate constants for the four isomers, the proximal configurations being favored over the distal ones. The switch from a concerted pathway at zero force to a sequential one occurs for a threshold force that is significantly higher $(\approx 2.4 \mathrm{nN})$ for the distal-exo adduct than for the other three $(\approx 1 \mathrm{nN})$, explaining its larger resistance to breaking and its almost inert character under tension. The switch is accompanied by the rupture of one of the two scissile bonds which leads to a twice smaller imaginary frequency of the transition state and an increase of the activation barrier, which then decreases for higher force strengths $(>3 \mathrm{nN})$ to become barrierless at a critical force value.
\end{abstract}




\section{Introduction}

Dynamic covalent bonds, like the archetypal furan-maleimide Diels-Alder (DA) adduct, are vastly used in synthetic chemistry and polymer science, and are now expanding into biology. ${ }^{1-}$ 3. Furan-maleimide adducts are characterized by dynamical covalent bonds that are longer and weaker than typical ones. The DA reactions on these adducts proceed via a low reaction barrier that leads to reversible reactions. In solution, the adducts need to be heated at high temperatures to break open. ${ }^{1}$ The retro-DA reaction can also take place at room temperature when the adduct is sonicated, thus subjected to mechanical forces. ${ }^{4,5}$ Four adducts with a different regio- and stereochemistry can be synthesized, namely the endo or exo configurations and proximal or distal geometries. These features make the furan-maleimide adducts attractive to design intelligent materials and logic devices driven by mechanical forces. Under thermal conditions, the reactivity is dictated by the stereochemistry, favoring the endo stereoisomers over the exo ones for bond breaking. ${ }^{6-8}$ Sonication bulk experiments by one of us have shown that unlike the thermal counterpart, the mechanical reactivity is mainly dependent on the regiochemistry. ${ }^{4}$ Remarkably, the thermally active distal-exo adduct was shown to be inert under sonication. Recently, Craig and co-workers probed the relative mechanical lability of stereoisomers of proximal DA adducts under sonication and found that the mechanical lability also depends on stereochemistry. ${ }^{5}$ Despite these important findings, a more complete and more quantitative picture of the effects of regio- and stereochemistry on the mechanics of DA adducts is clearly missing.

Beyond stabilizing intermediates and shifting chemical equilibria, due to the vector character of the force, mechanochemical reactions can follow specific pathways on the force modified potential energy surfaces (FM-PES), ${ }^{9}$ yielding products that may differ from those of nondirectional classical activation in solution (like thermal activation). ${ }^{9-12}$ Changes in the reaction mechanism upon applying an external force have been demonstrated in several polymer mechanophores with the support of theoretical simulations, ${ }^{13-15}$ i.e., in $2+2$ cyclo reversion of substituted 16-18, multicycle 19 and ladder type 20, 21 cyclobutane and in dimethylbenzocyclobutene ${ }^{22,23}$. Various 4+2 mecanophores have also be investigated ${ }^{4,5,24-30}$ as well as other functionally active systems. ${ }^{31,32}$ Single-molecule force spectroscopy (SMFS), which consists in trapping and stretching a single molecule between an atomic force microscopy (AFM) tip and a surface, has contributed to some developments in 
mechanochemistry. ${ }^{9,11}$ It has enabled the mechanical activation of covalent bonds, ${ }^{33-35}$ with applications in irreversible bond scission, ${ }^{10,23,31,36-42}$ and opening of mechanophores. ${ }^{23,43-51}$

Changes of mechanism under tension for retro Diels-Alder thermally allowed $4+2$ cyclo reactions have however been less systematically studied. 4, 5, 29, 30 Recently, a stepwise mechanism was evidenced upon the action of an electric field ${ }^{52}$, see also ref ${ }^{6}$ for a theoretical investigation. Here, we systematically modeled the response of the four DA adducts shown in Figure 1, proximal endo $\left(\mathrm{P}_{\text {endo }}\right)$, proximal exo $\left(\mathrm{P}_{\text {exo }}\right)$, distal endo $\left(\mathrm{D}_{\text {endo }}\right)$ and distal exo $\left(\mathrm{D}_{\text {exo }}\right)$, to an external force applied on the anchoring atoms used to tether them to a polymer linker (like the linkers used in ultrasound-induced mechanochemistry ${ }^{4}$ or in AFM-based SMFS ${ }^{53-57}$ ) using isometric $^{22,58}$ and isotensional ${ }^{47}$ approaches. For each force value, we characterized the strain energy redistribution using the JEDI method ${ }^{59}$ and the stationary points (equilibrium geometry and transition state (TS)). Our results concurrently show that the effect of the external force is to switch the mechanism of the retro (r)-DA from a concerted mechanism at zero force to a sequential one when a mechanical force is applied. The sequential path goes through an asynchronous TS leading to a stable intermediate where only one C-C bond is broken. This TS exhibits a slightly zwitterionic character. The switching of mechanism occurs at a rather sharp force value that is about $\approx 1 \mathrm{nN}$ for the two proximal adducts and the $\mathrm{D}_{\text {endo }}$ one, and at a larger value $(\approx 2.4 \mathrm{nN})$ for the Dexo one. The higher value of the switching explains the resilience of the $\mathrm{D}_{\text {exo }}$ adduct to bond breaking compared to the other three. This resilience is so high that at larger force value, it is the anchoring bond between $\mathrm{N}$ and $\mathrm{C}$ on the maleimide moiety that ruptures and not the second scissile bond. The switching of mechanism is confirmed by the change of the geometry of the TS and of its polarity, the decrease of its imaginary frequency by about a factor 2 and the localization of the frontier orbitals. We further identify the critical force for which the first bond breaking becomes barrierless using the approach of Makarov at al. ${ }^{60,61}$
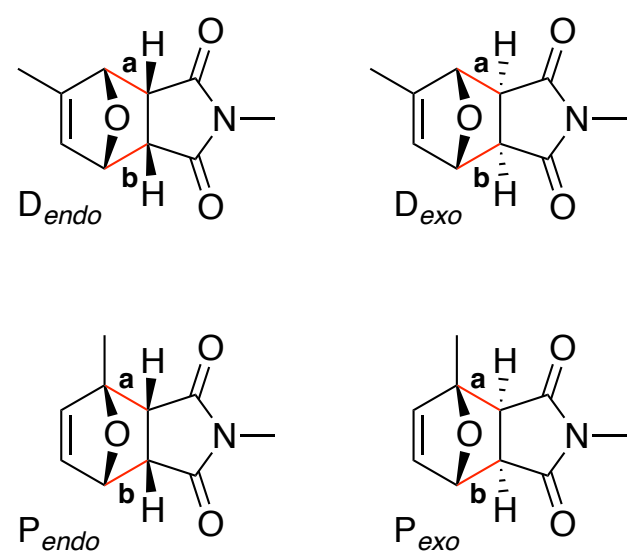
Figure 1 : The four furan/maleimide adducts investigated. They differ by their stereo (endo and exo) and regio (Distal and Proximal) character. The putative scissile bonds are shown in red and labeled ' $\mathbf{a}$ ' and ' $\mathbf{b}$ '. ' $\mathbf{a}$ ' is the bond along the shortest distance between the two methyl groups to which the pulling force is applied.

\section{Computational Methods}

Several theoretical approaches have been developed to provide understanding on the control of reactivity that can be achieved in covalent mechanochemistry using the directionality and the strength of the applied external force. Here we employ a quantum chemistry based static approach, which consists in analyzing the force modified potential energy surfaces (FMPES) computed within the Born and Oppenheimer approximation. The effect of the external force on the PES can be computed in an isometric or in an isotensional way. The isometric way consists in imposing as a constraint the distance between the two atoms that are pulled apart, here the two carbon atoms of the $\mathrm{CH}_{3}$ groups on the furan and on the maleimide moieties (see Figure 1 and Figure 2a) and relaxing the geometry for a scan of the constraint distances. This approach is called 'Geometry constraint simulate external force' (CoGEF). ${ }^{62}$ This is the approach adopted in ref. ${ }^{4}$ to simulate the response of the DA adducts. In CoGEF, the effect of the force is therefore modeled indirectly, through imposing a distance, $\mathrm{q}_{0}$, which is the controlled parameter. In the isotensional approach, forces in opposite directions are directly applied to the two carbon atoms. ${ }^{47}$ In this approach called 'External Force is Explicitly Included', (EFEI), the geometry is therefore relaxed directly for a scan of the force value, $\mathbf{F}_{0}$, which is the control parameter. It was shown by Marx et $\mathrm{al}^{47}$ that at stationarity, the $V_{\mathrm{EIEF}}$ potential, $V_{\mathrm{EFEI}}\left(\mathbf{F}_{0}\right)$ is the Legendre transform of the CoGEF one:

$$
V_{\mathrm{EFEI}}\left(\mathbf{F}_{0}\right)=V_{\mathrm{CoGEF}}\left(\mathbf{q}_{0}\right)-\mathbf{F}_{0} \cdot \mathbf{q}_{0}
$$

so that the state of mechanical equilibrium at a specified force, $\mathbf{F}_{0}$, is associated with a unique constrained distance $\mathbf{q}_{0}$. The EFEI method has the advantage to directly yield the Forcemodified Born -Oppenheimer PES. The relaxed geometry describes the effect of the specified force, $\mathbf{F}_{0}$, on the geometry. It also allows for a direct determination of the equilibrium 'reactant state (RS)' geometry, $\mathbf{q}_{\mathrm{RS}}\left(\mathbf{F}_{0}\right)$, and that of the transition state (TS), $\mathbf{q}_{\mathrm{TS}}\left(\mathbf{F}_{0}\right)$, under the specified force. ${ }^{12,47}$, see also ref. ${ }^{63}$ for a review of the different approaches. An approach similar to EFEI, the 'Enforced Geometry optimization on nuclei, EGO/N) was proposed simultaneously ${ }^{64}$ and extended to electrons. ${ }^{65}$ We compare below results obtained by the CoGEF and EFEI approaches using $\mathbf{F}_{0}=-\mathbf{F}_{0}^{\text {int }}=\nabla V\left(\mathbf{q}_{0}\right)$ where $\mathbf{F}_{0}^{\text {int }}$ is the internal restoring force. For a 
stationary point the relation between the constrained distance $\mathbf{q}_{0}$ and the corresponding force $\mathbf{F}_{0}$ is not linear. The EFEI method does not allow to determine a relaxed geometry after the bond rupture while the CoGEF one does. We use the EFEI approach below to compute transitions states, activation energies and rate constants because it is more straightforwardly related to AFM-based SMFS experiments since the force is the control parameter.

As can be seen from Figure 2a, each adduct has a different spatial configuration on which the external force projects in a specific way on several internal degrees of freedom, including the scissile bonds. In contrast to a thermal activation, the effect of the force does not only distinguish between endo/exo isomerism, it is geometry-dependent and thus, the four isomers correspond to four different responses upon mechanical activation.

The mechanochemical transduction of an applied force of a given strength and direction is determined by the force constants of the Hessian matrix which governs the redistribution of the strain energy on the internal degrees of freedom (bond length, angles, and dihedrals) and therefore by which bonds are more likely to rupture. Relying on the harmonic approximation implies that the analysis can only be applied at low and moderate forces, when the anharmonic effects are not too large. We analyze the redistribution of the strain energy using the JEDI (Judgement of Energy Distribution) approach. ${ }^{59,63,66-68}$ We base our analysis of the strain energy redistribution on the EFEI approach which, as discussed above, allows us to locate new stationary points as a function of the force in the FMPES and therefore is more straightforward for updating the Hessian. The strain energy at a given force $F$ in a given internal coordinate $q_{i}$ is given by :

$$
E_{i}^{F}=\left.\frac{1}{2} \sum_{j}^{M} \frac{\partial^{2} V(\mathbf{q})}{\partial q_{i} \partial q_{j}}\right|_{q=q_{0}} \Delta q_{i}^{F} \Delta q_{j}^{F}
$$

where the Hessian matrix, $\mathbf{H}=\Delta \mathbf{V}$, is transformed in redundant internal coordinates as in ref. ${ }^{69} . \mathbf{q}_{0}$ refers to the equilibrium geometry when no force is applied, $\Delta q_{i}^{F}=q_{i}^{F}-q_{i}^{0}$. The total harmonic strain energy is given by

$$
\Delta E_{\text {strain }}^{F}=\sum_{i}^{M} E_{i}^{F}=\left.\frac{1}{2} \sum_{i, j}^{M} \frac{\partial^{2} V(\mathbf{q})}{\partial q_{i} \partial q_{j}}\right|_{q=q_{0}} \Delta q_{i}^{F} \Delta q_{j}^{F}
$$

For the harmonic approximation to be meaningful, $\Delta E_{\text {strain }}^{F}$ should be comparable to the energy difference between the relaxed geometry under force and the one relaxed under the force constraint. The increment in stress energy obtained for each force interval up to the force 
considered, $F$, are added to obtain the total strain energy, $\Delta E_{\text {strain }}^{F}=\sum_{k=1}^{K} \Delta E_{\text {strain }}^{F_{k}}$ where $F=k d F$. We updated the molecular Hessian at every step, $\mathrm{d} F$ of JEDI, to better describe the energy landscape at larger displacements.

Applying an external force can either enhance or decrease the reactivity of targeted bonds. It is therefore of interest to determine the optimal force strength and direction to promote a desire outcome in a chemical reaction. ${ }^{60,70} 71-73$ At high force, the system can reach a very particular geometry that corresponds to a fold catastrophe ${ }^{74,75}$ where two stationary points, for example the reactant state and the transition state, coalesce. ${ }^{49,60,61,70-72,76,77}$ For this critical force value, $F_{c}$, the destabilized stable reactant state (RS) coalesces with a $1^{\text {st }}$ order transition state (TS) and undergoes a barrierless rupture. It this force value, the determinant of the Hessian matrix is zero and the eigenvector which corresponds to this zero eigenvalue provides the optimal direction of the force. ${ }^{70-72}$

Several algorithms have been proposed to locate this critical point. ${ }^{60,70} \mathrm{We}$ implemented the approach proposed by Makarov et al ${ }^{60,61,76}$ to analyze the FMPES around these critical points and determine the structure of the transition state in its vicinity. This algorithm has been shown to be very useful to analyze a broad range of chemical reactions including retroDA bond breaking. ${ }^{60,61,76}$

The algorithm consists in following the trajectory followed by a stationary point (either the RS or the TS) as the force is varied. The stationary points satisfy the equation

$$
\nabla \mathbf{V}=\mathbf{F}
$$

where $\nabla V$ is the gradient of the PES. If the force is increased by $d \mathbf{F}$, then the change in the critical configuration is given by

$$
\nabla \mathbf{V}(\mathbf{q}+d \mathbf{q})=\nabla \mathbf{V}(\mathbf{q})+\Delta \mathbf{V}(\mathbf{q}) d \mathbf{q}=\mathbf{F}+d \mathbf{F}
$$

where $\Delta \mathbf{V}(\mathbf{q})=\mathbf{H}\left(\mathbf{q}_{\mathbf{F}}\right)$ is the Hessian matrix corresponding to the FMPES at force $\mathbf{F}$. One therefore gets a path for the TS and a path for the RS respectively by integrating the following equation :

$$
\frac{d \mathbf{q}_{T S, R S}}{d F}=\mathbf{H}^{-1}\left(\mathbf{q}_{R S, T S}^{F}\right) \mathbf{e}
$$


q describes the molecular configuration of the RS or of the TS in the $3 \mathrm{~N}$-dimensional Cartesian space at the force strength $F, \mathbf{e}$ is the unit vector describing the direction of the pulling force in the $3 \mathrm{~N}$ space, $d \mathbf{F}=d F \mathbf{e}$ and $\mathbf{H}^{-1}$ is the pseudo inverse of the molecular Hessian matrix with rotational and translational degrees of freedom appropriately projected out. Eq (6) shows that the inverse of the Hessian matrix acts as a transformation matrix that connects two ForceModified PES (FMPES) for the specified direction of the force e. At the critical value of the force, $\mathbf{H}^{-1}$ diverges. The proximity to the fold catastrophe is located by following the eigenvalue spectrum of the Hessian. The trajectory of the TS and of the RS determine the reaction path of the mechanochemical transformation. We report such paths below for the four adducts. The force strength at the vicinity of the fold-catastrophe can be estimated with a precision that will depend mostly on the quality of the numerical method used to integrate Eq. (6) and the initial conditions. We found that Euler integrator, with a force step of 0.0002 a.u. performed well for our system. We implemented this algorithm in a Fortran program, the Hessian is obtained through calls to Q-Chem program. Using the zero-force RS and TS as initial conditions, we integrated Eq. (6) from each up to a point where they coalesce using for the vector $\mathbf{e}$ in Eq. (6) the pulling direction defined in Figure 2a. For these initial conditions, Eq. (6) lead to the same stationary points (reactant state and transition states) as the EFEI approach. The advantage of using Eq. (6) is that one can more straightforwardly determine the point corresponding to the critical force where these two stationary points coalesce.

The determination of the equilibrium geometries of the ground electronic state at zero force and under force were carried out using Density Functional Theory with the CAM-B3LYP functional including the D3 Grimme correction and the triple zeta Pople basis set 6$311++\mathrm{G}(2 \mathrm{df}, 2 \mathrm{p})$. The Q-Chem program ${ }^{78}$ was used throughout. The free energy differences are computed within the harmonic-rigid rotor approximation, by means of a vibrational analysis. For more computational demanding tasks like the determination of the enforced reaction path, we use the smaller basis set $6-31 \mathrm{G}^{* *}$.

\section{Results and discussion}

\section{Mechanical response}

The direction of the applied force is shown in Figure $2 \mathrm{a}$. The results for the isometric CoGEF and isotensional EFEI pulling protocols are shown in Figure $2 \mathrm{~b}$ and $\mathrm{c}$, respectively, where the 
total energy is plotted as a function of the control distance in panel $b$ and as function of the control force in panel c.

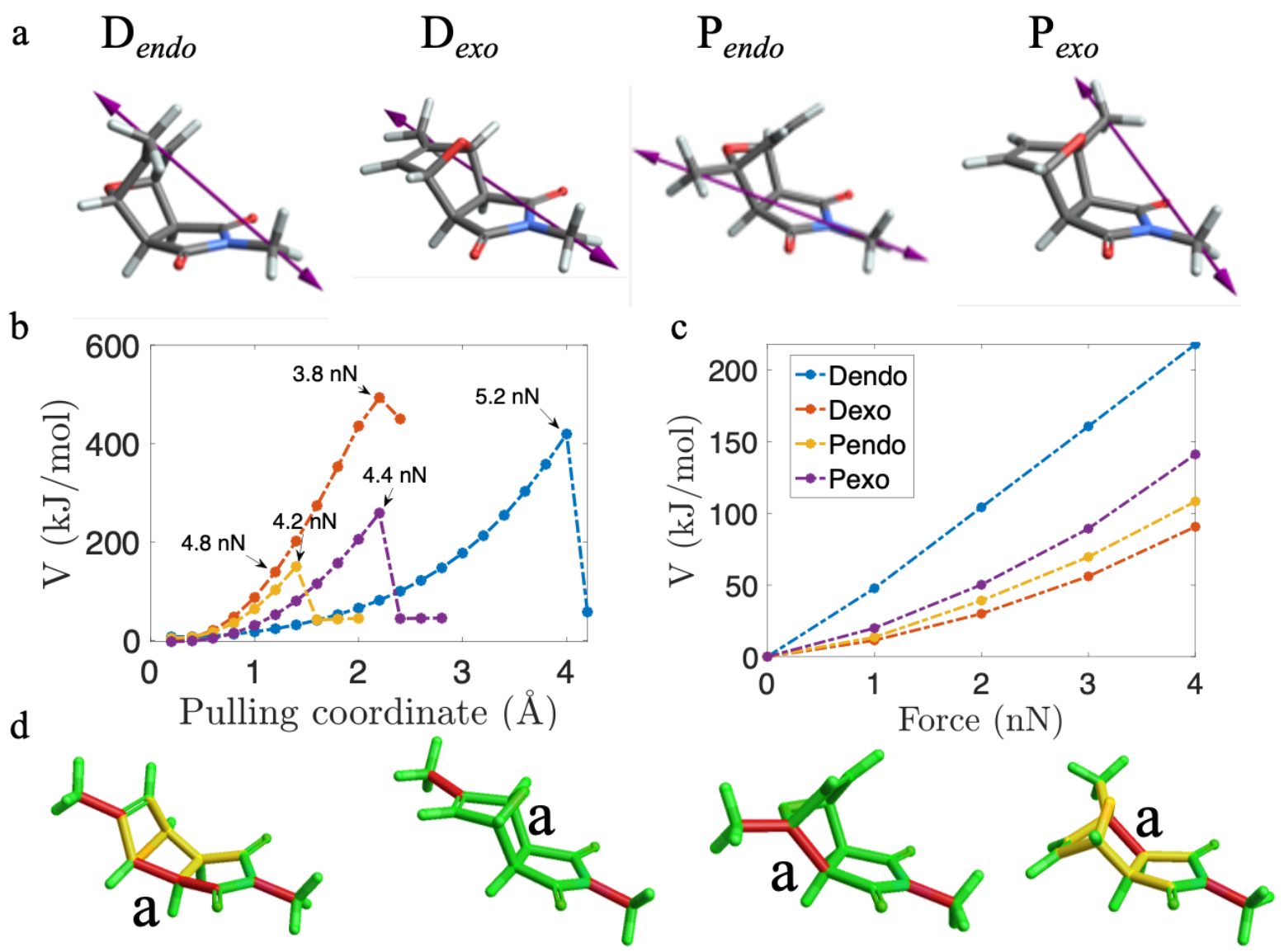

Figure $2:$ a) Equilibrium geometry of the adducts at zero force and direction of the pulling force. b) and c) Change in the potential energy of the furan-maleimide adducts with the external force simulated by isometric (b) and isotensional (c) approaches. b) COGEF energy, the pulling coordinate refers to the distance between the atoms being pulled, see a). c) EFEI energies obtained for increasing values of the force applied on the same atoms in the direction of the pulling coordinate. In (b) the forces at equilibrium with a constraint just before breaking are computed using $\mathbf{F}_{0}=-\mathbf{F}_{0}^{\text {int }}=\nabla V\left(\mathbf{q}_{0}\right)$. In the case of the $\mathrm{D}_{\text {exo }}$ adduct, no rupture was observed at the scissile bond. Instead the stationary point obtained is a TS for the $\mathrm{N}-\mathrm{CH}_{3}$ rupture on the maleimide moiety. The value of the force is $4.8 \mathrm{~nm}$ at an elongation that corresponds to the rupture of the $\mathrm{P}_{\text {endo }}$ adduct. d) Geometries and colored view of the redistribution of the strain energy on the bonds of the adducts computed for an external force of $4 \mathrm{nN}$. Regions in red hold more than $10 \mathrm{~kJ} / \mathrm{mol}$ of stress energy, those in yellow, between $5-10 \mathrm{~kJ} / \mathrm{mol}$ and the ones in green, exhibit less than $5 \mathrm{~kJ} / \mathrm{mol} .4 \mathrm{nN}$ is close to the forces computed at the last CoGEF 
point before the rupture of the adducts that break apart (see Figure $2 b$ ). The strain energies are reported in Table 1. The scissile bond marked ' $a$ ' corresponds to the bonds on which the force shown in (a) has the largest projection. See SI for more details on the analysis of the energy redistribution.

As can be seen from Figure $2 \mathrm{~b}$ and $\mathrm{c}$, the responses of the two distal adducts to tension are the most extreme. The $\mathrm{D}_{\text {endo }}$ adduct responds very smoothly to the stretching and undergoes the largest change in potential energy when the external force is applied. The rupture of the scissile bond occurs for a force of $5.2 \mathrm{nN}$ (Figure 2b). This implies that the mechanophore embedded in the polymer in the $\mathrm{D}_{\text {endo }}$ configuration could be stretched significantly since the external force remains in equilibrium with the internal restoring forces. On the other hand, upon pulling, the $\mathrm{D}_{\text {exo }}$ adduct, previously characterized as very resilient to the rupture, ${ }^{4}$ does not break apart into furan and maleimide fragments. Instead, the $\mathrm{N}-\mathrm{CH}_{3}$ bond of maleimide side is broken. This explains the rather steep rise of the potential energy observed in Figure $2 b$. By following the value of the external force that corresponds to the controlled distance using $\mathbf{F}_{0}=-\mathbf{F}_{0}^{\text {int }}=\nabla V\left(\mathbf{q}_{0}\right)$, we see that the adduct reaches force values of the same order of magnitude as the $\mathrm{P}_{\text {endo }}$ adduct, but instead of undergoing the breaking of a scissile bond between the maleimide and the furan, it follows an alternative path until the $\mathrm{N}-\mathrm{CH}_{3}$ bond ruptures, as is shown below. This means that the CoGEF curve for the $\mathrm{D}_{\text {exo }}$ adduct, to satisfy the geometrical constraint, is deviating from the EFEI path shown in Figure 2c which allows to follow the response in the pulling direction for larger values of the force. In summary, an external force applied to $\mathrm{D}_{\text {exo }}$ through a controlled distance (Figure $2 \mathrm{~b}$ ) does not promote the rupture of the mechanophore at the scissile bonds, but instead promotes the rupture at a bond that links the adduct to the polymer chain. This kind of fragmentation does not produce two stable fragments, and therefore a constrained optimization fails to locate a minimum under the controlled distance as in the CoGEF protocol. Instead, the stationary point of the CoGEF protocol corresponds to a TS. The response of the adducts in a proximal configuration $\left(\mathrm{P}_{\text {exo }}\right.$ and $\left.\mathrm{P}_{\text {endo }}\right)$ is intermediate between those of the distal adducts. The response of $\mathrm{P}_{\text {endo }}$ is closer to that of the $\mathrm{D}_{\text {exo }}$ but one of the scissile bond breaks for a rather small elongation. On the other hand, in the $\mathrm{P}_{\text {exo }}$ configuration, a longer pulling distance can be applied until the breaking occurs, but this adduct is not as resistant as $\mathrm{D}_{\text {endo. }}$.

For a same stereochemistry character, we therefore observe a difference between the adducts according to the regiochemistry: the distal adduct tends to be more resistant than the proximal 
one. This could be a consequence of a more general effect due to the specific directions of the applied external force used to pull the mechanophore apart shown in Figure 2a. The stereochemistry can still be determinant since for the same regio character, the endo configuration is found to favor the rupture. It is well-known that, at thermal conditions, the reactivity of the endo isomer is higher than the exo counterpart, which is a consequence of a smaller energy barrier. Our results on thermal activation are reported in the SI. They fully confirm these results and are in agreement with previous computational and experimental results ${ }^{4}$.

Table 1. Redistribution of the stress energy among the internal modes in the furan-maleimide adducts in equilibrium with a $4 \mathrm{nN}$ force. Energies are given in $\mathrm{kJ} / \mathrm{mol}$. The bond ' $\mathbf{a}$ ' is shown in Figure 2d.

\begin{tabular}{|l|c|c|c|c|c|}
\hline & $\Delta E_{\text {strain }}^{F}$ & $\Delta E_{\text {strain in bond }}^{F}$ & \% in stretching & \% in bending & $\%$ in torsion \\
\hline $\mathbf{D}_{\text {endo }}$ & 217.9 & 7.4 & 9 & 21 & 70 \\
\hline $\mathbf{D}_{\text {exo }}$ & 90.6 & 1.3 & 16 & 24 & 60 \\
\hline $\mathbf{P}_{\text {endo }}$ & 108.3 & 13.9 & 22 & 25 & 53 \\
\hline $\mathbf{P}_{\text {exo }}$ & 141.1 & 13.0 & 29 & 24 & 47 \\
\hline
\end{tabular}

In order to get further insights the activation of the internal degrees of freedom of the adducts upon pulling, we analyzed the redistribution of the stress energy using the JEDI approach (see Methods section) implemented on the equilibrium geometries obtained with the isotensional (EFEI) approach at $4 \mathrm{nN}$. The computed values of the stress energy in the different internal coordinates are reported in Table 1 and shown graphically in Figure $2 \mathrm{~d}$. The stress energy computed for a pulling force of $4 \mathrm{nN}$ is redistributed in specific ways in each adduct. The distal adducts contain significantly less strain in the scissile bonds than proximal ones and the opposite is true for the torsions. The strain energies in angles are quite similar for the four adducts. This agrees with the results shown in Figure $2 \mathrm{~b}$ and $\mathrm{c}$ where distal adducts are seen to be more resistant to the external perturbation. Remarkably, the strain energy in the $\mathrm{D}_{\text {exo }}$ adduct is the smallest of all the compounds and this is because this adduct barely elongates at an external force of $4 \mathrm{nN}$. For the $\mathrm{D}_{\text {exo }}$ adduct, the force primarily projects onto the bonds that link the mechanophore to the polymer chain, leaving the core quite unchanged compared to the zero-force state (Figure $2 \mathrm{a}$ ). In the $\mathrm{D}_{\text {endo }}$ adduct, the stress energy is considerably higher than in the other three and redistributed more uniformly over all bonds. As a result, the molecule is 
very distorted at this force strength as can be seen from Figure 2 d. For this adduct and the proximal ones, the total stress energy is consistent with the level of distortion of the molecular geometry that can be judged by comparing the geometries shown in Figure 2a (zero force) and $2 \mathrm{~d}(\mathrm{a} 4 \mathrm{nN}$ force).

Because of the asymmetry in the direction of the pulling force (see Figure 2a), the scissile bond ' $a$ ' that projects better on the force direction, elongates more than the other scissile bond. In the case of the proximal adducts, a major part of the strain is concentrated in the ' $a$ ' bond which explains why these adducts break at the lower force values. Although the forces around the discontinuity point in CoGEF are similar, the elongation in the $\mathrm{P}_{\text {endo }}$ adduct is smaller than in the $\mathrm{P}_{\text {exo }}$ and that is why the strain is smaller too. The $\mathrm{D}_{\text {endo }}$ mechanophore is still resilient to a force of $4 \mathrm{nN}$, but there is a considerable amount of strain in the scissile bond ' $a$ ' compared to the rest of the molecule. Although there is more relative strain into the scissile bond ' $a$ ' compared to the $\mathrm{D}_{\text {endo }}$ adduct, the scissile bonds of the $\mathrm{D}_{\text {exo }}$ adduct scarcely participate into the stress energy redistribution (Figure $2 \mathrm{~d}$ ). Conversely, at this stress energy the $\mathrm{N}-\mathrm{CH}_{3}$ bond is activated and this explains why the rupture occurs in that location as noted before.

\section{Effect of the external force on the reaction mechanism}

For a thermal activation, rDA reactions follow a concerted mechanism that involves the two scissile bonds simultaneously and the reaction path involves a unique transition state (TS). The TS stereochemistry obeys that of the adducts, so that there is an endo and exo TS. We show in Figure 3 the changes induced by a $0.5 \mathrm{nN}$ and a $4 \mathrm{nN}$ force on the geometry of the TS and on the localization of the frontier MO's for the $\mathrm{D}_{\text {exo }}$ (Figure $3 \mathrm{a}$ ) and the $\mathrm{P}_{\text {endo }}$ (figure $3 \mathrm{~b}$ ). 


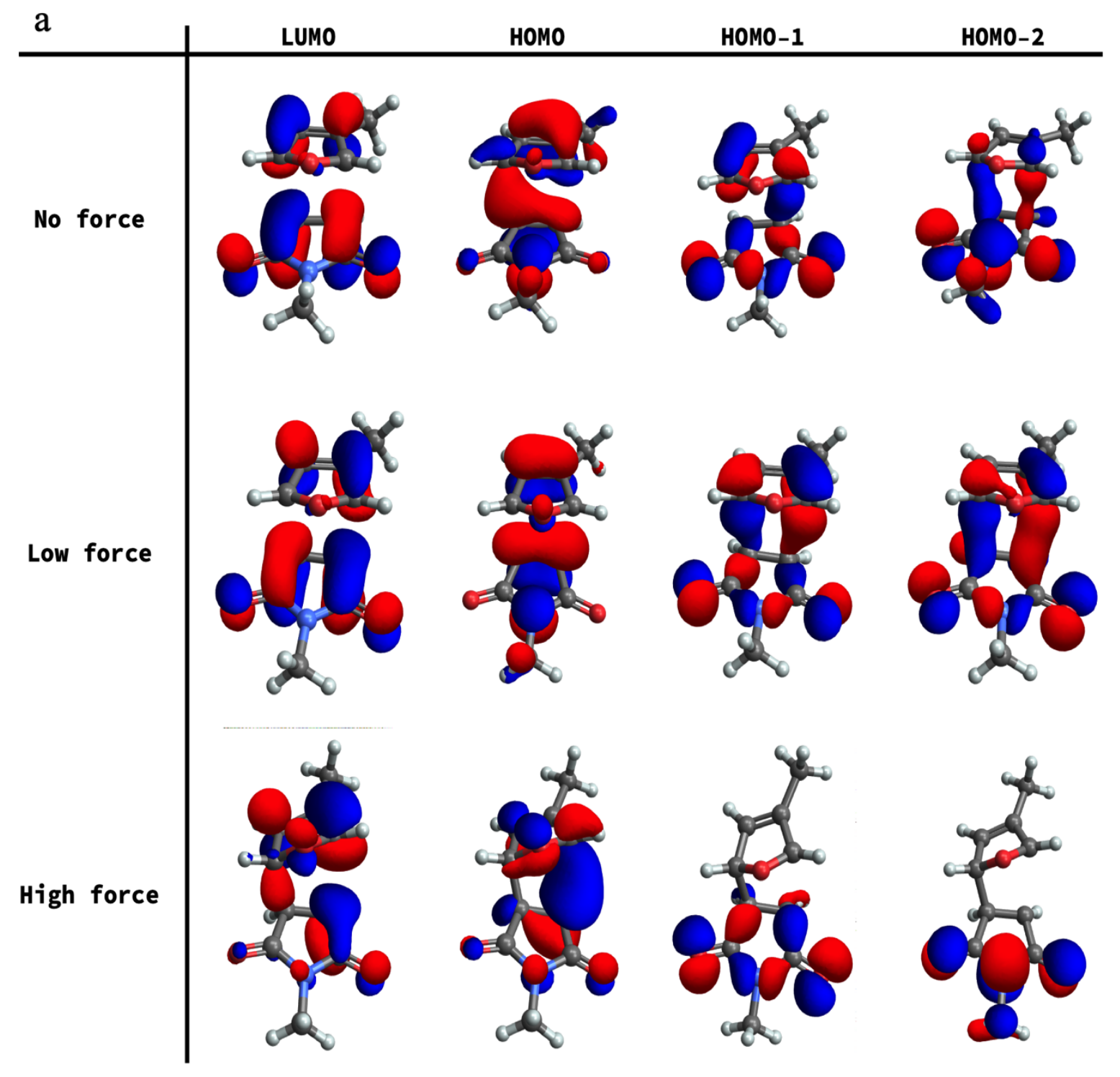




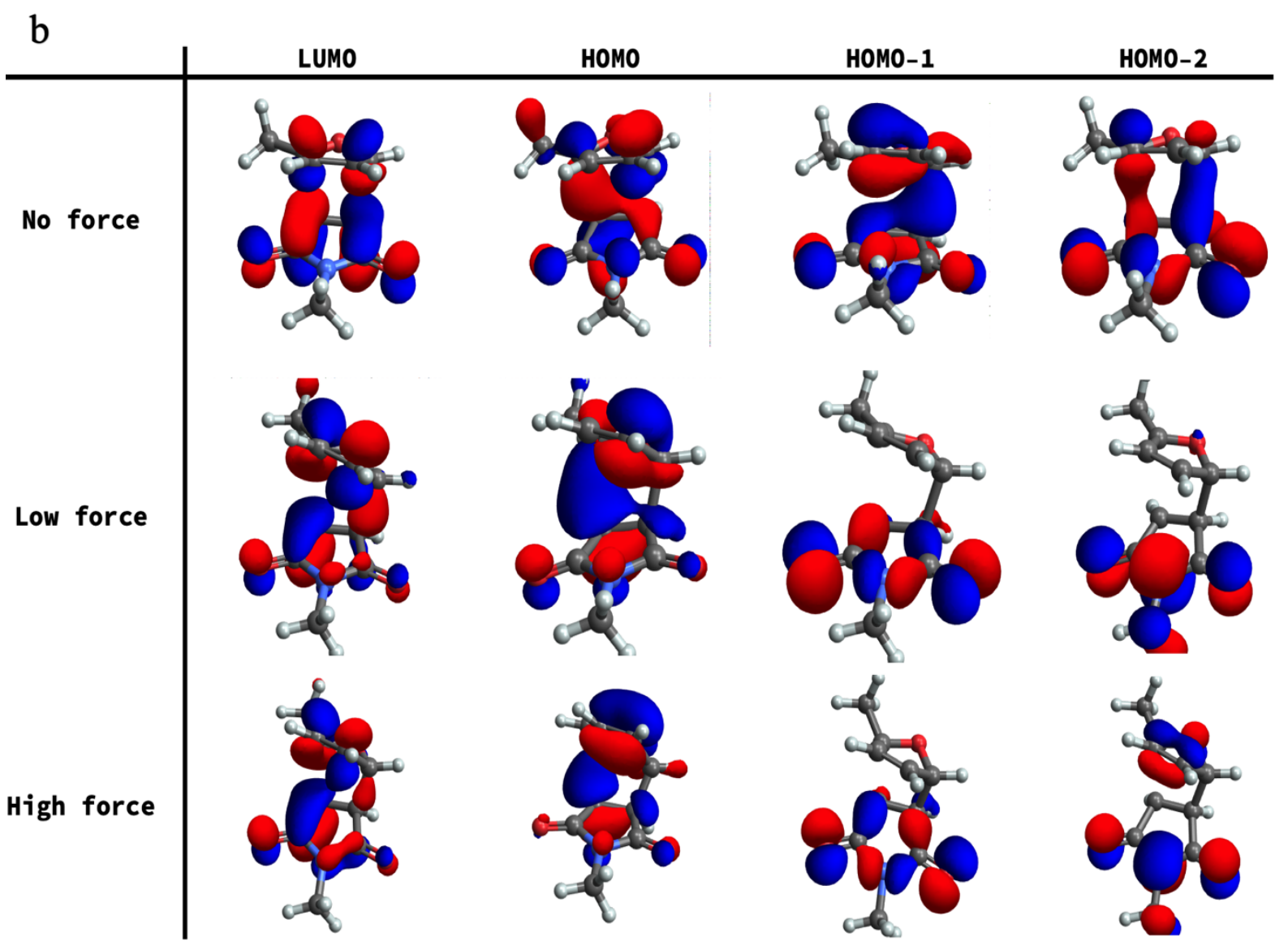

Figure 3. Geometries of $\mathrm{D}_{\text {exo }}$ (a) and $\mathrm{P}_{\text {endo }}$ (b) TS for furan/maleimide rDA reaction at no force, low force $(0.5 \mathrm{nN})$, and high force $(4 \mathrm{nN})$. Isocontours $\left(0.02 / \AA^{3}\right)$ of the frontier MO's are also shown. The geometries and MO's of the $\mathrm{D}_{\text {endo }}$ and $\mathrm{P}_{\text {endo }}$ adducts are reported in Figure S4 and S5 of the SI.

As can be seen from the geometries shown in Figure 3 for a force of $4 \mathrm{nN}$, the external force induces a significant geometry change of the TS in the $\mathrm{nN}$ regime, while for sub- $\mathrm{nN}$ forces, as expected for such a small perturbation, the geometry of the TS differs less from the zero force one. The change in geometry is a consequence of the rearrangement of the system to reach a stationary point under the force constraint. Because of the asymmetry of the direction of the force with respect to the two scissile bonds, as the force is increasing, one scissile bond (bond a) becomes longer than the other one. A similar change was observed in the equilibrium geometry of the reactant state at $4 \mathrm{nN}$, see Figure $2 \mathrm{~d}$.

The electronic structure also responds to this change in geometry, and the changes are very striking as can be seen from the isocontours of the frontier MO's plotted in Figure 3. Small forces do not significantly perturb the energies and spatial localization of the MO's, and thus 
the TS remains similar to the zero-force case, which means that the concerted mechanism is being preserved. However, as the force increases, the structural distortion is evident and thus the MO's are distorted too. At high force, there is significantly less electronic density localized in the region of the scissile bonds for the HOMO-1 and HOMO-2 orbitals which are localized on the maleimide part. This trend already appears at low force for the $\mathrm{P}_{\text {endo }}$ adduct (Figure $3 \mathrm{~b}$ ). Consequently, a one-step concerted mechanism is less likely to occur and the reaction pathway is likely to be different from the one followed at zero-force. The MO's of the TS of the $\mathrm{P}_{\text {exo }}$ and the $\mathrm{D}_{\text {endo }}$ adducts follow similar trends as can be seen from Figures S3 and S4 of the SI.

Such a switch of mechanism has been reported in a polar solvent for the $4+2$ Claisen rearrangement. ${ }^{79}$ For this reaction, in solution, the TS is found to evolve to a zwitterionic form while it is diradicalar in the gas phase. Computational studies of the effect of a static external electric field on the endo and exo reaction paths of a similar adduct, the cyclopentadienemaleic anhydride adduct, showed that the direction of the applied electric field can affect differently the endo and exo pathways. ${ }^{6}$ The activation or inhibition of the rDA by electric field of different orientations is shown to be due to the fact that the field can stabilize or destabilize the reactant and the TS differently and in opposite directions, which leads to higher or lower reaction barriers depending on its orientation. These authors also reported that the effect can be larger in a polar solvent due to the larger geometry change resulting from the solvent effects. A clear zwitterionic transition state is characterized in a polar solvent when the direction of the field is parallel to the scissile bonds. Such a switch in the reaction pathway under the perturbation of an external electrical field was recently confirmed experimentally, ${ }^{52}$ by subjecting the adducts tethered to a polymethyl methacrylate chain (PMMA) to high voltages in a graphene transistor.

Here we report a switch to a sequential pathway under a mechanical external force. Since the charge separation can only arise from the geometry changes induced by the external force, the zwitterionic character of the TS and of the stable intermediate are less pronounced than those observed under the effect of an external electric field in a polar solvent. But the external force induces a large difference in the length of the $\mathrm{C}-\mathrm{C}$ bonds, which is of the order of $0.9 \AA$ at the force where the transition takes place.

The consequences that this switch in the TS have into the mechanism of the reaction can also be assessed by the analysis of the thermochemistry under pulling. Having determined the geometry of the TS and of the adduct (called reactant state (RS) from now) for each force value, we can compute the energetic barrier for the rDA reaction of the four furan-maleimides adducts in gas phase at room temperature. This allows us to quantify the effect of the force on the 
magnitude of the barrier which is important for assessing the reactivity of the system using the Eyring-Polanyi TS theory. ${ }^{80,81}$

The variation of the barrier height under pulling is shown in Figure 4a, where two different regimes can clearly be distinguished according to the value of the applied external force. The free energy barriers and the variation of the rate constants under force are reported in the SI. For forces in the sub-nN regime, the barrier energy increases, which makes the dissociation of the adduct less probable than at zero force. The four adducts become more resistant to the rupture. However, for larger forces a decrease of the barrier is observed. For the two proximal and the $\mathrm{D}_{\text {endo }}$ adducts, the barrier drops quickly. For these three adducts, the reactivity is enhanced at forces larger $\approx 2 \mathrm{nN}$ while it is suppressed at lower ones. On the other hand, the barrier does not drop and remains quite high for the $\mathrm{D}_{\text {exo }}$ adduct, which confirms the mechanical resilience of this mechanophore to break under force, as observed experimentally ${ }^{4}$ and discussed above. The force suppresses the reactivity of the $\mathrm{D}_{\text {exo }}$ adduct.

The change in the molecular structure of the TS, in addition to the reduction of the barrier height, suggests a crossover of the mechanism similar to the one previously reported by Meir et al computing the effect of an external electric field as the perturbation ${ }^{6}$ and recently observed experimentally. ${ }^{52}$ The mechanism switch between the two regimes is also reflected in the value of the imaginary frequency of the TS, shown in Figure $4 \mathrm{~b}$. A dramatic decrease of the imaginary frequency takes place at $\approx 1 \mathrm{nN}$ for the $\mathrm{D}_{\text {exo }}$ adduct. This value corresponds to the force for which the barrier shown in Figure 4a reaches its maximum. The imaginary frequency corresponds to the negative eigenvalue of the Hessian matrix which characterizes the topology of the PES around the first-order saddle point. When the adduct is distorted along the direction of the eigenvector that corresponds to the imaginary frequency, there is no restoring force taking it back to a stable equilibrium and such a distortion is said to be an unstable perturbation. The switch of mechanism suggested by the sharp decrease (by a factor $\approx 2$ ) of the value of the imaginary frequency is supported by the structural rearrangement of the TS shown in Figure 3 in which one scissile bond is much stronger (and shorter) than the other one. The difference in bond length of the two scissile bonds is plotted in Figure 4c.

The TS that corresponds to a sequential mechanism is more polarized than the concerted one, as can be seen from the value of the permanent moment plotted in Figure $4 \mathrm{~d}$ and the value of the partial charge on each fragment reported in panels e and $\mathrm{f}$. However, in the case of a change driven by a mechanical force, as expected, the polarity increase is less pronounced than the one reported when the adducts are perturbed by an external electric field. ${ }^{6}, 52$ Here, the charge transfer results from the Born-Oppenheimer relaxation of the electronic density that adjusts to 
the equilibrium position of the nuclei under constraint. It does not exceed a few tens of electron charge $|\mathrm{e}|$.
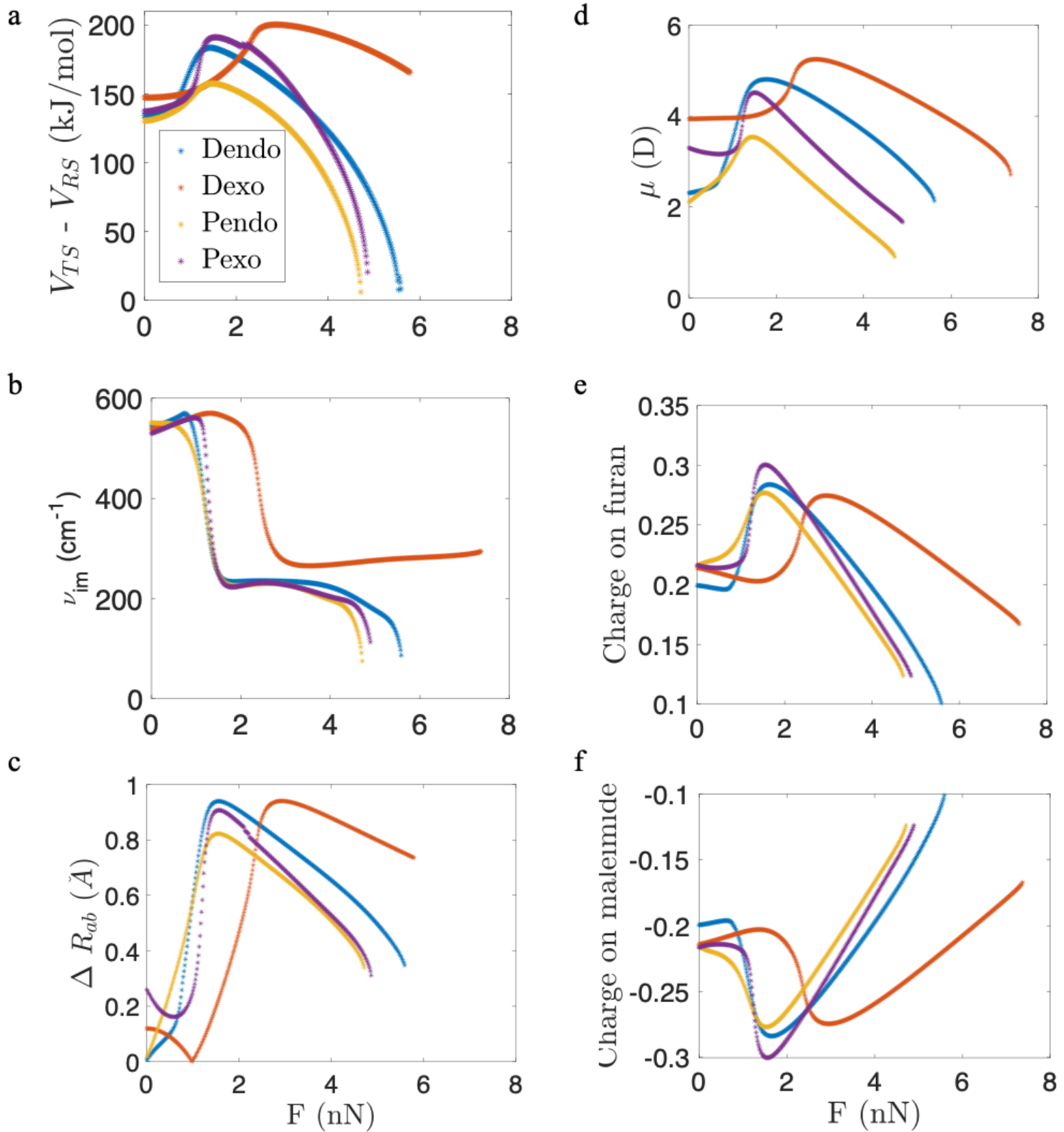

Figure 4a. a) Barrier height in $\mathrm{kJ} / \mathrm{mol}$ of each furan-maleimide adduct under force. The reaction barrier for the rDA reactions increases with force strength in the sub-nN regime, except in the case of $\mathrm{D}_{\text {exo }}$ for which the sharp increase occurs at forces larger than $2 \mathrm{nN}$ and then decreases slightly. For the three other adducts, the barrier height drops dramatically at larger forces. This behavior corresponds to a suppression of reactivity for lower forces and an enhancing at larger ones. This change can be related to the switch to a sequential mechanism at larger force. b) Imaginary frequencies of the TS states computed for the same range of forces. 
For all the adducts except $\mathrm{D}_{\text {exo }}$, around a $1 \mathrm{nN}$ force, the imaginary frequency of the TS drops by a factor 2 . This point corresponds to the onset of the switching from a concerted to a sequential mechanism. For $\mathrm{D}_{\text {exo }}$, as expected, this switch occurs at a larger force. c) Difference in the bond lengths of the two scissile bonds. d) Dipole moment of the transition state. Its value increases by about a factor 2 at $\approx 1 \mathrm{nN}$. This change reflects both the geometry change that can be seen in Figure 4 and also a charge transfer from the furan to the maleimide moiety, as shown in panel e (partial charge on the furan moiety) and panel $\mathrm{f}$ (partial charge on the maleimide moiety)

\section{Critical force and force-induced reaction path}

Catastrophe theory predicts ${ }^{76}$ that for a specific direction of the force, the FMPES reaches a singularity where the negative eigenvalue of the TS and one of positive eigenvalues of the RS become zero. This point on the FMPES is called fold-catastrophe, because at this value of the force, the geometries of TS and RS coalesce. This also means that at this point, the force aligns with an eigenvector of the Hessian matrix and a distortion along this direction does not meet any resistance from the system. In these conditions, the molecule becomes mechanically unstable since a small force $d F$ along this direction can easily induce bond breaking.

The fold-catastrophe is the most typical case found in mechanochemistry. However other kinds of scenarios can occur, see ref. ${ }^{60}$, for an extensive discussion on the topic. In particular it is possible that the RS coalesces with TS associated to another reaction than the one targeted. In this case, the molecule will break in a different position and another reaction will occur.

Locating this singularity of the PES relying on the EFEI method is a very complex task, since it is very difficult to converge a geometry optimization at the vicinity of the fold-catastrophe point. As explained in the Method section above, ${ }^{61}$ all the force-displaced stationary points obtained through an EFEI optimization satisfy Eq. (6). The numerical integration of Eq. (6) starting from the TS and the RS zero force equilibrium geometries and increasing force values in the pulling direction until a zero-eigenvalue is found for both stationary points can be used to assess whether the direction of the force applied to the furan-maleimide adducts will eventually lead to the rupture of the scissile bonds.

Figure 5 shows the force-induced path followed by the four adducts as the force is increased up to the critical value. It contains all the force-displaced stationary points (FDSP) that can be located through a conventional EFEI optimization The fold catastrophe occurs when the RS and TS coincide. At this point of the FMPES, the force is the maximum possible that can be 
exerted onto the system and its value is the threshold for the mechanical barrierless reaction. The results shown in Figure 5 for the RS state are fully consistent with those shown in Figure 2c using the isotensional approach.
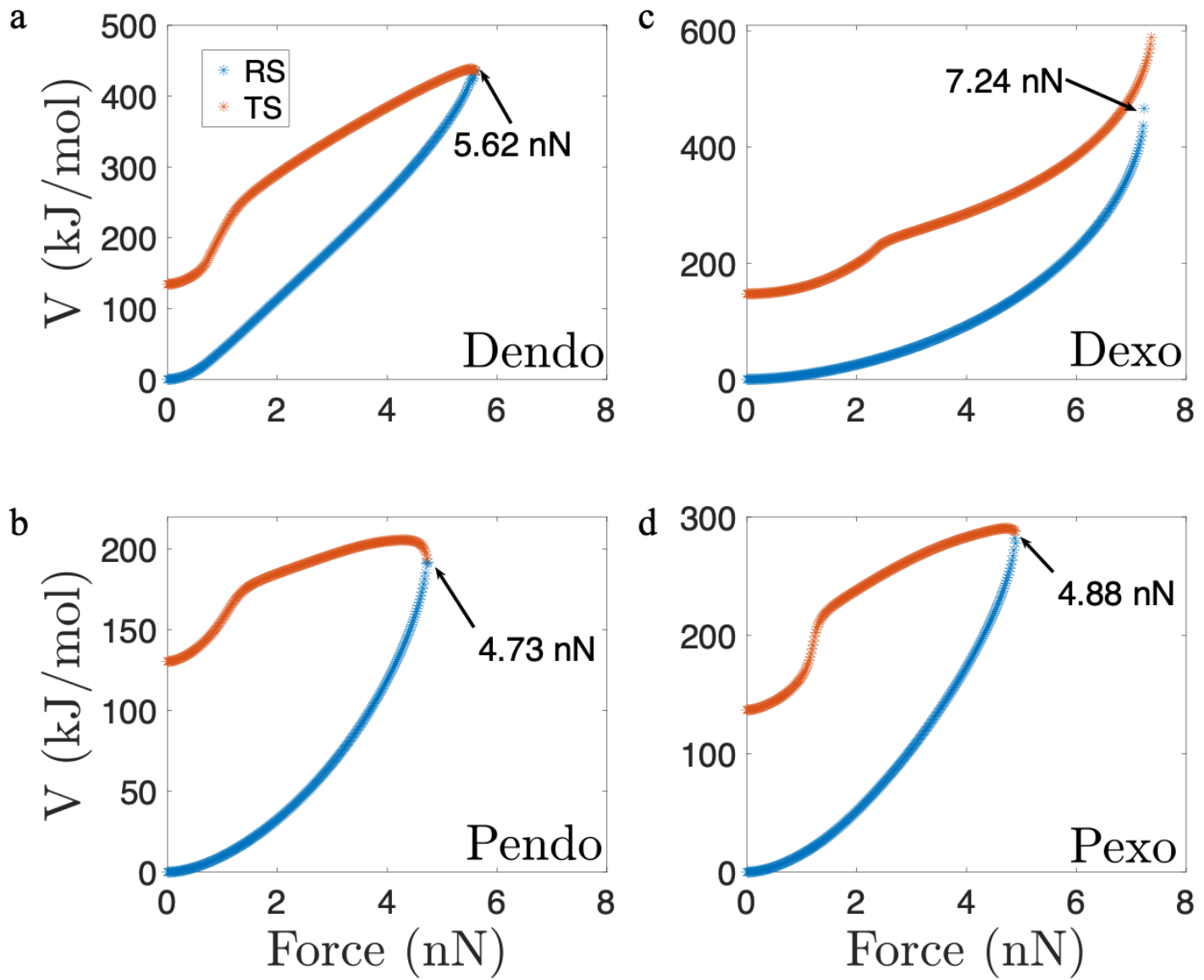

Figure 5 Potential energy as a function of the pulling force applied on each adduct (see Figure 2a for the direction), computed at the geometries obtained by integrating Eq. (6). For the $\mathrm{D}_{\text {endo }}$ (a), $\mathrm{P}_{\text {endo }}(\mathrm{b})$ and $\mathrm{P}_{\text {exo }}(\mathrm{d})$ adducts, both the TS and RS energies initially increase with the force but at the vicinity of the catastrophe point the energy of the TS decreases until it coalesces with the RS. The curve of the RS energy is monotonically increasing, but the TS has an inflexion point around $1 \mathrm{nN}$, which corresponds to the region of transition between a concerted and a stepwise dissocation, identified above on the basis of the height of the barrier and the value of the imaginary frequency of the TS (see Figure 4). On the other hand, the TS and RS of the $\mathrm{D}_{\text {exo }}$ adduct (panel c) reach an asymptote at higher force, where a highly unstable geometry (second order saddle point) is located.

Upon integration using Eq. (6), the RS of all the adducts except the $\mathrm{D}_{\text {exo }}$ coalesce with the TS of the rDA reaction. Proximal adducts reach the critical point around similar forces (4.73 and 
$4.88 \mathrm{nN}$, for endo and exo isomers, respectively). The $\mathrm{D}_{\text {endo }}$ adduct requires a larger force (5.62 $\mathrm{nN}$ ) which confirms the larger resilience of this adduct to the external force identified above based on the analysis of the strain energy, the TS geometry and MO's, and of the height of the reaction barrier. These values are in very good agreement with the computed rupture force of the isometric CoGEF protocol (figure $2 \mathrm{~b}$ ) : $4.2 \mathrm{nN}$ for $\mathrm{P}_{\text {endo }}, 4.4 \mathrm{nN}$ for $\mathrm{P}_{\text {exo }}$ and $5.2 \mathrm{nN}$ for $\mathrm{D}_{\text {endo. }}$. The difference can be understood from the fact that in the CoGEF protocol, since the distance is the control parameter, one cannot vary the force in a systematic way.

The Dexo adduct was experimentally characterized as resilient to break. ${ }^{4}$ The analysis based on the critical force provides an explanation for such a behavior. As shown in Figure S6 of the SI, the RS coalesces with a TS corresponding to the rupture of the $\mathrm{N}-\mathrm{CH}_{3}$ bond in maleimide part, not with the TS of the rDA reaction. The system is destabilized by the force, and instead of following the same path as the rest of the adducts in the FMPES, an alternative one is pursued. The RS coalesces with a first-order saddle point and the TS coalesces with a second order-saddle point. The force at which it happens (around $7 \mathrm{nN}$ ) is quite large compared to the other adducts. Therefore, the mechanophore in a Dexo configuration does not undergo the rupture of the scissile bonds in agreement with the experimental results. ${ }^{4}$

\section{Conclusions}

The effect of a pulling external force was investigated for four Diels-Alder adducts which differ by their regio- and stereochemistry. Since the force projects differently on the scissile bonds in each adduct, this allows us to explore the effect of the direction of the force on the adduct response. Our computational results agree with experimental results of ultrasound-based. 4 They explain why the proximal adducts are more reactive under the force constraint than distal counterparts, which is a consequence of a better projection of the force on the scissile bonds. In the case of the $\mathrm{D}_{\text {exo }}$ adduct, the resilience to undergo the rDA reaction, also previously observed in experiments, ${ }^{4}$ is confirmed by our theoretical computations. The analysis of the redistribution of the strain in the $\mathrm{D}_{\text {exo }}$ adduct showed that the external force affects preferentially the bonds that connect the mechanophore to the polymer chain, while the scissile bonds are barely being distorted. This means that a rupture of the $\mathrm{D}_{\text {exo }}$ adduct through a rDA reaction, giving back the furan and maleimide species, is very unlikely. For the $\mathrm{D}_{\text {endo }}$ adduct, the CoGEF and EFEI analysis of the effect of the external force on the geometry and potential energies of the adduct show that the mechanophore in this configuration can resist a long 
elongation without opposing a large restoring force. The analysis of the redistribution of the stress energy shows that it is redistributed over the entire molecule and not in specific coordinates involving the scissile bonds as for the rest of the adducts. The order followed by the breaking of the adducts in CoGEF isometric protocol $\left(\mathrm{P}_{\text {endo }} \approx \mathrm{P}_{\text {exo }}<\mathrm{D}_{\text {endo }}\right)$ is in agreement with the one obtained following the force displaced stationary points and correlates with the analysis of the redistribution of the strain energy.

We show that for low force values the reactivity is inhibited by the stretching of the adduct and the reaction barrier becomes higher than at zero force. However, at larger forces (typically $>3$ $\mathrm{nN}$ ), the barrier decreases and the reactivity of the rDA is enhanced. When we follow the force displaced stationary points, eventually, at a critical value of the force, the reaction becomes barrierless and the molecule breaks without opposing to the external perturbation. This is the value of the force for which the TS and RS coalesce in a singularity of the Force-Modified PES. The nature of the resilience of $\mathrm{D}_{\text {exo }}$ to the rupture was attributed in an atypical catastrophe, that coalesced the RS to a different TS, which conducts to the breaking of another bond of the adduct.

Finally, a crossover from a concerted to a stepwise mechanism was identified. The forces at which this switch occurs were estimated at about $1 \mathrm{nN}$ for the two proximal adducts and for the $\mathrm{D}_{\text {endo }}$ one, and at $2.4 \mathrm{nN}$ for the $\mathrm{D}_{\text {exo }}$ one. The abrupt changes observed in the localization of the frontier molecular orbitals, of the imaginary frequencies of the TS, as well as in the difference in length of the two scissile bonds and in the values of the dipole moment, suggest a transition to a sequential mechanism, with a more polar, asynchronous TS.

These computational results are an outstanding support for the interpretation of AFM-based single-molecule force spectroscopy experiments that are currently in progress to obtain a detailed picture of the mechanical stability of the adducts, including the time they can resist to a constant force.

Acknowledgements

ARC, CECI

References

1.Kotha, S.; Banerjee, S., Recent developments in the retro-Diels-Alder reaction. RSC Advances 2013, 3, 7642-7666.

2.Zhang, Z. P.; Rong, M. Z.; Zhang, M. Q., Polymer engineering based on reversible covalent chemistry: A promising innovative pathway towards new materials and new functionalities. Progress in Polymer Science 2018, 80, 39-93. 
3.Chakma, P.; Konkolewicz, D., Dynamic Covalent Bonds in Polymeric Materials. Angewandte Chemie International Edition 2019, 58, 9682-9695.

4.Stevenson, R.; De Bo, G., Controlling Reactivity by Geometry in Retro-Diels-Alder Reactions under Tension. Journal of the American Chemical Society 2017, 139, 16768-16771.

5.Wang, Z.; Craig, S. L., Stereochemical effects on the mechanochemical scission of furanmaleimide Diels-Alder adducts. Chemical Communications 2019, 55, 12263-12266.

6.Meir, R.; Chen, H.; Lai, W.; Shaik, S., Oriented Electric Fields Accelerate Diels-Alder Reactions and Control the endo/exo Selectivity. ChemPhysChem 2010, 11, 301-310.

7.Rulíšek, L.; Šebek, P.; Havlas, Z.; Hrabal, R.; Čapek, P.; Svatoš, A., An Experimental and Theoretical Study of Stereoselectivity of Furan-Maleic Anhydride and Furan-Maleimide Diels-Alder Reactions. The Journal of Organic Chemistry 2005, 70, 6295-6302.

8.Ogawa, A.; Fujimoto, H., Reexamination of orbital interactions in Diels-Alder reactions. Tetrahedron Letters 2002, 43, 2055-2057.

9.Polymer Mechanochemistry. Springer: 2015; Vol. 369.

10.Akbulatov, S.; Tian, Y.; Huang, Z.; Kucharski, T. J.; Yang, Q.-Z.; Boulatov, R., Experimentally realized mechanochemistry distinct from force-accelerated scission of loaded bonds. Science 2017, 357, 299-303.

11.Garcia-Manes, S.; Beedle, A. E. M., Steering chemical reactions with force. Nature Chemistry 2017, 1.

12.Ribas-Arino, J.; Marx, D., Covalent Mechanochemistry: Theoretical Concepts and Computational Tools with Applications to Molecular Nanomechanics. Chemical Reviews 2012, $112,5412-5487$.

13.Izak-Nau, E.; Campagna, D.; Baumann, C.; Göstl, R., Polymer mechanochemistry-enabled pericyclic reactions. Polymer Chemistry 2020, 11, 2274-2299.

14.Akbulatov, S.; Boulatov, R., Experimental Polymer Mechanochemistry and its Interpretational Frameworks. ChemPhysChem 2017, 18, 1422-1450.

15.Tian, Y.; Kucharski, T. J.; Yang, Q.-Z.; Boulatov, R., Model studies of force-dependent kinetics of multi-barrier reactions. Nature Communications 2013, 4, 2538.

16.Zhang, H., et al., Multi-modal mechanophores based on cinnamate dimers. Nature Communications 2017, 8, 1147.

17.Kean, Z. S.; Niu, Z.; Hewage, G. B.; Rheingold, A. L.; Craig, S. L., Stress-Responsive Polymers Containing Cyclobutane Core Mechanophores: Reactivity and Mechanistic Insights. Journal of the American Chemical Society 2013, 135, 13598-13604.

18.Kryger, M. J.; Ong, M. T.; Odom, S. A.; Sottos, N. R.; White, S. R.; Martinez, T. J.; Moore, J. S., Masked Cyanoacrylates Unveiled by Mechanical Force. Journal of the American Chemical Society 2010, 132, 4558-4559.

19.Liu, Y.; Holm, S.; Meisner, J.; Jia, Y.; Wu, Q.; Woods Toby, J.; Martinez Todd, J.; Moore Jeffrey, S., Flyby reaction trajectories: Chemical dynamics under extrinsic force. Science 2021, 373, 208-212.

20.Horst, M.; Yang, J.; Meisner, J.; Kouznetsova, T. B.; Martínez, T. J.; Craig, S. L.; Xia, Y., Understanding the Mechanochemistry of Ladder-Type Cyclobutane Mechanophores by Single Molecule Force Spectroscopy. Journal of the American Chemical Society 2021, 143, 12328 12334.

21.Chen, Z.; Zhu, X.; Yang, J.; Mercer, J. A. M.; Burns, N. Z.; Martinez, T. J.; Xia, Y., The cascade unzipping of ladderane reveals dynamic effects in mechanochemistry. Nature Chemistry 2020, 12, 302-309.

22.Hickenboth, C. R.; Moore, J. S.; White, S. R.; Sottos, N. R.; Baudry, J.; Wilson, S. R., Biasing reaction pathways with mechanical force. Nature 2007, 446, 423-427. 
23.Wang, J.; Kouznetsova, T. B.; Niu, Z.; Ong, M. T.; Klukovich, H. M.; Rheingold, A. L.; Martinez, T. J.; Craig, S. L., Inducing and quantifying forbidden reactivity with singlemolecule polymer mechanochemistry. Nature Chemistry 2015, 7, 323-327.

24.McFadden, M. E.; Robb, M. J., Force-Dependent Multicolor Mechanochromism from a Single Mechanophore. Journal of the American Chemical Society 2019, 141, 11388-11392.

25.Potisek, S. L.; Davis, D. A.; Sottos, N. R.; White, S. R.; Moore, J. S., Mechanophore-Linked Addition Polymers. Journal of the American Chemical Society 2007, 129, 13808-13809.

26.Robb, M. J.; Kim, T. A.; Halmes, A. J.; White, S. R.; Sottos, N. R.; Moore, J. S., Regioisomer-Specific Mechanochromism of Naphthopyran in Polymeric Materials. Journal of the American Chemical Society 2016, 138, 12328-12331.

27.Wang, Z.; Ma, Z.; Wang, Y.; Xu, Z.; Luo, Y.; Wei, Y.; Jia, X., A Novel Mechanochromic and Photochromic Polymer Film: When Rhodamine Joins Polyurethane. Adv Mater 2015, 27, 6469-74.

28.Wang, T.; Zhang, N.; Dai, J.; Li, Z.; Bai, W.; Bai, R., Novel Reversible Mechanochromic Elastomer with High Sensitivity: Bond Scission and Bending-Induced Multicolor Switching. ACS Applied Materials \& Interfaces 2017, 9, 11874-11881.

29.Hu, X.; McFadden, M. E.; Barber, R. W.; Robb, M. J., Mechanochemical Regulation of a Photochemical Reaction. J Am Chem Soc 2018, 140, 14073-14077.

30.Kida, J.; Imato, K.; Goseki, R.; Aoki, D.; Morimoto, M.; Otsuka, H., The photoregulation of a mechanochemical polymer scission. Nat Commun 2018, 9, 3504.

31.Beedle, A. E. M.; Lezamiz, A.; Stirnemann, G.; Garcia-Manyes, S., The mechanochemistry of copper reports on the directionality of unfolding in model cupredoxin proteins. Nature Communications 2015, 6, 7894.

32.Nixon, R.; De Bo, G., Three concomitant $\mathrm{C}-\mathrm{C}$ dissociation pathways during the mechanical activation of an N-heterocyclic carbene precursor. Nature Chemistry 2020, 12, 826-831.

33.Duwez, A.-S.; Cuenot, S.; Jérôme, C.; Gabriel, S.; Jérôme, R.; Rapino, S.; Zerbetto, F., Mechanochemistry: targeted delivery of single molecules. Nature Nanotechnology 2006, 1, 122-125.

34.Beyer, M. K.; Clausen-Schaumann, H., Mechanochemistry: The Mechanical Activation of Covalent Bonds. Chemical Reviews 2005, 105, 2921-2948.

35.Kersey, F. R.; Yount, W. C.; Craig, S. L., Single-Molecule Force Spectroscopy of Bimolecular Reactions: System Homology in the Mechanical Activation of Ligand Substitution Reactions. Journal of the American Chemical Society 2006, 128, 3886-3887.

36.Wiita, A. P.; Ainavarapu, S. R. K.; Huang, H. H.; Fernandez, J. M., Force-dependent chemical kinetics of disulfide bond reduction observed with single-molecule techniques. Proceedings of the National Academy of Sciences 2006, 103, 7222-7227.

37.Zheng, P.; Arantes, G. M.; Field, M. J.; Li, H., Force-induced chemical reactions on the metal centre in a single metalloprotein molecule. Nature Communications 2015, 6, 7569.

38.Grandbois, M., How Strong Is a Covalent Bond? Science 1999, 283, 1727-1730.

39.Zheng, P.; Li, H., Highly Covalent Ferric-Thiolate Bonds Exhibit Surprisingly Low Mechanical Stability. Journal of the American Chemical Society 2011, 133, 6791-6798.

40.Lee, J.-H.; Nam, J.-M.; Jeon, K.-S.; Lim, D.-K.; Kim, H.; Kwon, S.; Lee, H.; Suh, Y. D., Tuning and Maximizing the Single-Molecule Surface-Enhanced Raman Scattering from DNATethered Nanodumbbells. ACS Nano 2012, 6, 9574-9584.

41.Pill, M. F.; East, A. L. L.; Marx, D.; Beyer, M. K.; Clausen-Schaumann, H., Mechanical Activation Drastically Accelerates Amide Bond Hydrolysis, Matching Enzyme Activity. Angewandte Chemie International Edition 2019, 58, 9787-9790.

42.Schütze, D.; Holz, K.; Müller, J.; Beyer, M. K.; Lüning, U.; Hartke, B., Pinpointing Mechanochemical Bond Rupture by Embedding the Mechanophore into a Macrocycle. Angewandte Chemie International Edition 2015, 54, 2556-2559. 
43.Hansmann, M. M.; Melen, R. L.; Rudolph, M.; Rominger, F.; Wadepohl, H.; Stephan, D. W.; Hashmi, A. S. K., Cyclopropanation/Carboboration Reactions of Enynes with B(C6F5)3. Journal of the American Chemical Society 2015, 137, 15469-15477.

44.May, P. A.; Moore, J. S., Polymer mechanochemistry: techniques to generate molecular force via elongational flows. Chemical Society Reviews 2013, 42, 7497.

45.Li, J.; Nagamani, C.; Moore, J. S., Polymer Mechanochemistry: From Destructive to Productive. Accounts of Chemical Research 2015, 48, 2181-2190.

46.Chen, Z.; Mercer, J. A. M.; Zhu, X.; Romaniuk, J. A. H.; Pfattner, R.; Cegelski, L.; Martinez, T. J.; Burns, N. Z.; Xia, Y., Mechanochemical unzipping of insulating polyladderene to semiconducting polyacetylene. Science 2017, 357, 475-479.

47.Ribas-Arino, J.; Shiga, M.; Marx, D., Understanding Covalent Mechanochemistry. Angewandte Chemie International Edition 2009, 48, 4190-4193.

48.Klukovich, H. M.; Kean, Z. S.; Ramirez, A. L. B.; Lenhardt, J. M.; Lin, J.; Hu, X.; Craig, S. L., Tension Trapping of Carbonyl Ylides Facilitated by a Change in Polymer Backbone. Journal of the American Chemical Society 2012, 134, 9577-9580.

49.Ribas-Arino, J.; Shiga, M.; Marx, D., Mechanochemical Transduction of Externally Applied Forces to Mechanophores. Journal of the American Chemical Society 2010, 132, 10609-10614. 50.Pill, M. F.; Holz, K.; Preußke, N.; Berger, F.; Clausen-Schaumann, H.; Lüning, U.; Beyer, M. K., Mechanochemical Cycloreversion of Cyclobutane Observed at the Single Molecule Level. Chemistry - A European Journal 2016, 22, 12034-12039.

51.Li, H.; Walker, G. C., Twist and Shout: Single-Molecule Mechanochemistry. ACS Nano 2017, 11, 28-30.

52.Yang, C., et al., Electric field-catalyzed single-molecule Diels-Alder reaction dynamics. Science Advances 2021, 7, eabf0689.

53.Lussis, P.; Svaldo-Lanero, T.; Bertocco, A.; Fustin, C.-A.; Leigh, D. A.; Duwez, A.-S., A single synthetic small molecule that generates force against a load. Nature Nanotechnology 2011, 6, 553-557.

54.Van Quaethem, A.; Lussis, P.; Leigh, D. A.; Duwez, A.-S.; Fustin, C.-A., Probing the mobility of catenane rings in single molecules. Chemical Science 2014, 5, 1449.

55.Sluysmans, D.; Zhang, L.; Li, X.; Garci, A.; Stoddart, J. F.; Duwez, A.-S., Viologen Tweezers to Probe the Force of Individual Donor-Acceptor $\pi$-Interactions. Journal of the American Chemical Society 2020, 142, 21153-21159.

56.Sluysmans, D.; Lussis, P.; Fustin, C.-A.; Bertocco, A.; Leigh, D. A.; Duwez, A.-S., RealTime Fluctuations in Single-Molecule Rotaxane Experiments Reveal an Intermediate Weak Binding State during Shuttling. Journal of the American Chemical Society 2021, 143, 23482352.

57.Devaux, F.; Li, X.; Sluysmans, D.; Maurizot, V.; Bakalis, E.; Zerbetto, F.; Huc, I.; Duwez, A.-S., Single-molecule mechanics of synthetic aromatic amide helices: Ultrafast and robust non-dissipative winding. Chem 2021, 7, 1333-1346.

58.Beyer, M. K., The mechanical strength of a covalent bond calculated by density functional theory. The Journal of Chemical Physics 2000, 112, 7307-7312.

59. Stauch, T.; Dreuw, A., A quantitative quantum-chemical analysis tool for the distribution of mechanical force in molecules. Journal of Chemical Physics 2014, 140, 134107-134107.

60.Avdoshenko, S. M.; Makarov, D. E., Finding mechanochemical pathways and barriers without transition state search. The Journal of Chemical Physics 2015, 142, 174106.

61.Avdoshenko, S. M.; Makarov, D. E., Reaction Coordinates and Pathways of Mechanochemical Transformations. The Journal of Physical Chemistry B 2016, 120, 1537 1545 .

62.Beyer, M. K., The mechanical strength of a covalent bond calculated by density functional theory. Journal of Chemical Physics 2000. 
63.Stauch, T.; Dreuw, A., Predicting the Efficiency of Photoswitches Using Force Analysis. Journal of Physical Chemistry Letters 2016, 7, 1298-1302.

64.Wolinski, K.; Baker, J., Theoretical predictions of enforced structural changes in molecules. Molecular Physics 2009, 107, 2403-2417.

65.Wolinski, K.; Baker, J., Geometry optimization in the presence of external forces: a theoretical model for enforced structural changes in molecules. Molecular Physics 2010, 108, $1845-1856$.

66.Stauch, T.; Dreuw, A., On the use of different coordinate systems in mechanochemical force analyses. Journal of Chemical Physics 2015, 143, 074118-074118.

67.Stauch, T.; Dreuw, A., Advances in Quantum Mechanochemistry: Electronic Structure Methods and Force Analysis. American Chemical Society: 2016; Vol. 116, pp 14137-14180.

68.Stauch, T.; Dreuw, A., Quantum Chemical Strain Analysis For Mechanochemical Processes. Accounts of Chemical Research 2017, 50, 1041-1048.

69.Peng, C.; Ayala, P. Y.; Schlegel, H. B.; Frisch, M. J., Using redundant internal coordinates to optimize equilibrium geometries and transition states. Journal of Computational Chemistry 1996, 17, 49-56.

70.Bofill, J. M.; Ribas-Ariño, J.; García, S. P.; Quapp, W., An algorithm to locate optimal bond breaking points on a potential energy surface for applications in mechanochemistry and catalysis. The Journal of Chemical Physics 2017, 147, 152710.

71.Quapp, W.; Bofill, J. M., Mechanochemistry on the Müller-Brown surface by Newton trajectories. International Journal of Quantum Chemistry 2018, 118, e25522.

72.Quapp, W.; Bofill, J. M., Reaction rates in a theory of mechanochemical pathways. Journal of Computational Chemistry 2016, 37, 2467-2478.

73.Wolinski, K., Exploring Potential Energy Surface with External Forces. Journal of Chemical Theory and Computation 2018, 14, 6306-6316.

74.Gilmore, R., Catastrophe Theory for Scientists and Engineers. Wiley: New York, 1981.

75. Wales, D. J., A Microscopic Basis for the Global Appearance of Energy Landscapes. Science 2001, 293, 2067.

76.Konda, S. S. M.; Avdoshenko, S. M.; Makarov, D. E., Exploring the topography of the stress-modified energy landscapes of mechanosensitive molecules. The Journal of Chemical Physics 2014, 140, 104114.

77.Quapp, W.; Bofill, J. M., Comment on "Exploring Potential Energy Surface with External Forces". Journal of Chemical Theory and Computation 2020, 16, 811-815.

78. Shao, Y., et al., Advances in molecular quantum chemistry contained in the Q-Chem 4 program package. Molecular Physics 2015, 113, 184-215.

79.Cramer, C. J.; Truhlar, D. G., What causes aqueous acceleration of the Claisen rearrangement? Journal of the American Chemical Society 1992, 114, 8794-8799.

80.Laidler, K. J.; King, M. C., Development of transition-state theory. The Journal of Physical Chemistry 1983, 87, 2657-2664.

81.Levine, R. D., Molecular Reaction Dynamics. Cambridge University Press: Cambridge, 2005. 